\section{Desigualdade social no adoecimento e morte por câncer de boca e orofaríngeo no Município de São Paulo, Brasil: 1997 a 2008}

\author{
Social inequality in morbidity and mortality from \\ oral and oropharyngeal cancer in the city of \\ São Paulo, Brazil: 1997-2008
}

1 Universidade Federal do Rio Grande do Norte, Natal, Brasil.

2 Secretaria da Agricultura do Estado de São Paulo, São Paulo, Brasil.

3 Faculdade de Saúde Pública, Universidade de São Paulo, São Paulo, Brasil.

Correspondência M. A. F. Ferreira

Universidade Federal do Rio Grande do Norte.

Av. Rui Barbosa 1257, Bloco

A, apto. 204, Natal, $R N$

59056-300, Brasil.

angelaf@ufrnet.br

\section{Abstract}

Occupation of geographical space is historically determined by the socioeconomic model and the dynamism of its social, political, and ideological relations. This study aimed to evaluate the spatial distribution of morbidity and mortality from cancer of the mouth and oropharynx and related socioeconomic variables in the city of São Paulo, Brazil, from 1997 to 2008. Data were collected from the Population-Based Cancer Registry and the Program for Improvement of Mortality Data (PRO-AIM) and were georeferenced using Terraview and GeoDa. The theoretical framework for evaluating the results was the work of Milton Santos. The incidence rates showed spatial autocorrelation with a Global Moran index of 0.226 and mortality rates of 0.337. Incidence of cancer of the mouth and oropharynx did not show a well-defined spatial pattern in the city of São Paulo, but mortality rates were highly unequal, concentrating the lowest rates in the central area of the city, which is wealthier and economically less unequal.

Oropharyngeal Neoplasms; Social Inequity; Geography
Maria Angela Fernandes Ferreira 1

Murilo Novaes Gomes 2

Fernanda Alessandra Silva Michels 3

Aldo Aluísio Dantas 1

Maria do Rosário Dias de Oliveira Latorre 3

\section{Introdução}

Os estudos com base nos dados de incidência e mortalidade por câncer de boca e orofaríngeo devem ser constantemente atualizados no sentido de subsidiar gestores e planejadores na área da saúde, com novas informações sobre a sua frequência e distribuição. Especialmente no Município de São Paulo, Brasil, onde as taxa de mortalidade $1,2,3$ e incidência ${ }^{4}$ se situam entre uma das mais elevadas no mundo, tanto no sexo masculino quanto no feminino.

As altas taxas de incidência são atribuídas a fatores comportamentais como o consumo de tabaco 5 , álcool 6 e determinados hábitos dietéti$\cos 7$. Agentes infecciosos como o Papilomavírus também são citados como um dos responsáveis pelo desenvolvimento da doença ${ }^{8}$. Além desses agentes etiológicos, a mudança da estrutura populacional, com o aumento da esperança de vida e o maior acesso ao serviço de saúde, por meio de tratamentos específicos e programas de rastreamento para a detecção precoce da lesão ${ }^{9}$, são considerados algumas das razões pelas elevadas taxas da doença na atualidade.

Da mesma forma, fatores socioeconômicos, frequentemente, são apontados como determinantes importantes na incidência e mortalidade por câncer de boca e orofaríngeo, tanto nos estudos individuados 10 como nos ecológicos 11,12,13. Para a análise do contexto socioeconômico dos 
indivíduos ou dos grupos populacionais, os indicadores mais usados são aqueles construídos baseando-se em dados de renda, ocupação, escolaridade, longevidade, além de outros. No elenco de tais indicadores destacam-se o Índice do Desenvolvimento Humano (IDH), utilizado pela Organização Mundial da Saúde (OMS) para aferição da qualidade de vida, refletindo as condições socioeconômicas de uma dada população, e o índice de Gini, para avaliação do efeito da desigualdade/iniquidade social.

Um método importante e bastante ilustrativo para execução desses estudos é o georreferenciamento, pois é possível observar a distribuição espacial das taxas de câncer e correlacionar com os dados socioeconômicos. Isso porque o uso pleno do território como forma de análise das vulnerabilidades e dos determinantes à saúde pressupõe a identificação de objetos geográficos, seu uso pela população e sua importância para os fluxos das pessoas e materiais 14 .

Pensar considerando o território e o lugar é se contrapor a uma visão setorial que caracteriza as políticas públicas e o planejamento tradicional. Pensar sob a ótica do território significa analisar a complexidade, a totalidade, a complementaridade, a inter-relação que existe entre as coisas que precisamos para viver. Falar em estado de saúde é também falar em estado dos lugares, das regiões, da cidade, do bairro, do país. Mesmo sabendo que a totalidade é uma realidade fugaz que se desfaz e se refaz, é a realidade total que devemos procurar compreender. Estamos sempre diante de uma sociedade em movimento e isto nos coloca face a face a uma questão: como apreendê-la? Fazer um inventário de todas as características de um fenômeno ou de um indivíduo é uma tarefa insolúvel, já nos alertava Durkheim 15.

Os estudos acerca do câncer de boca e orofaríngeo no Município de São Paulo, publicados na literatura apresentam limitações na interpretação do efeito do espaço sobre esta patologia, na medida em que carecem de uma análise totalizante da realidade. Para uma melhor compreensão do efeito espacial sobre as doenças é imprescindível se ter em mente que o território é o local onde se processa um determinado modo de produção e suas relações sociais, políticas e ideológicas, com todas elas exercendo uma influência decisiva nas localizações 16 .

Nesse sentido, o resgate das teorias globalizantes de Milton Santos se faz necessário para nortear a análise espacial da mortalidade e incidência de câncer de boca e sua correlação com indicadores socioeconômicos no Município de São Paulo, no período de 1997-2008, objetivo deste trabalho.

\section{Metodologia}

\section{Desenho, local e população de estudo}

Estudo ecológico realizado no Município de São Paulo, que é a maior cidade do Brasil, com extensão de $1.530 \mathrm{~km}^{2}, 11.253 .503$ habitantes (Instituto Brasileiro de Geografia e Estatística. Cidades@. http://www.ibge.gov.br/cidadesat/topwindow. htm?1, acessado em 24/Ago/2011) e está localizada na região sudeste. O município é dividido em 96 distritos que apresentam fortes disparidades socioeconômicas, sendo que aqueles que se situam na região central são ricos e desenvolvidos, e os periféricos, em geral, com problemas de infraestrutura e indicadores sociais inferiores à média da cidade (Fundação Sistema Estadual de Análise de Dados. http:/ / www.seade.gov.br, acessado em 20/Jul/2011).

\section{Coleta de dados}

Os dados referem-se ao período de 1997-2008. Os dados de incidência de câncer orofaríngeo foram obtidos no Registro de Câncer de Base Populacional (Secretaria Municipal de Saúde. http://ww2.prefeitura.sp.gov.br/cgi/deftohtm. exe?secretarias/saude/TABNET/CA/cancer.def, acessado em 08/Jun/2011) e os de mortalidade na base de dados do Programa de Aprimoramento das Informações de Mortalidade - PRO-AIM (Secretaria Municipal de Saúde. http://www. prefeitura.sp.gov.br/cidade/secretarias/saude/ tabnet/mortalidade/index.php?p=6529, acessado em 08/Jun/2011). Foi definido câncer orofaríngeo aqueles compreendidos entre os códigos C-00 e C-10 (câncer de lábio, língua, gengiva, palato e úvula, mucosa oral, vestíbulo da boca, área retromolar, glândulas salivares maiores, amígdala e orofaríngeo), bem como áreas não especificadas da boca (C-14.8) da Classificação Internacional de Doenças 10a Revisão - CID-10 (http://www.bulas.med.br/cid-10, acessado em 05/Mai/2011).

Análise dos dados: A base cartográfica digital do município foi obtida na Secretaria Municipal de Planejamento, composta por diversas camadas possibilitando seu uso em softwares de Sistemas de Informação Geográfica (SIG). As taxas de incidência e mortalidade de câncer orofaríngeo (número de casos/população residente no distrito x 100 mil habitante/ano) foram calculadas e ajustadas pelo método direto usando a população padronizada mundial como referência 17 . O número de casos utilizado no cálculo das taxas de incidência foi de 9.593 e, nos de mortalidade, 4.426 óbitos. As análises foram realizadas utilizando os 94 distritos, pois para estabilização dos 
dados, dois distritos da região sul (Parelheiros e Marsilac) e dois da norte (Anhanguera e Perus) foram unificados.

As informações sociodemográficas foram coletadas e calculadas com base nos dados do Censo Demográfico de 2000 . O coeficiente de Gini é a medida de desigualdade da distribuição da renda de uma determinada população, que teoricamente varia de 0 (zero desigualdade) - em que todos receberiam a mesma parcela da renda total do distrito - para 1 (máxima desigualdade) - na qual uma família receberia o total de toda a renda do distrito. O IDH é obtido pela média aritmética simples de três subíndices referentes às dimensões longevidade, educação e renda. Esse índice não considera apenas fatores econômicos para medir o desenvolvimento de um país ou mesmo de um município, posto que agrega, na execução do cálculo, os fatores longevidade e educação embutidos no índice com peso igual ao da renda per capita, ajustada pelo poder de compra.

A partir das taxas encontradas foi realizado o georreferenciamento por distrito, usando-se o software Terraview 4.0.0 (Instituto Nacional de Pesquisas Espaciais; http://www.dpi.inpe.br/ terraview), no qual foram criados os mapas de incidência, mortalidade, IDH e Gini. Nos mapas criados, a intensidade dos níveis de cinza está relacionada diretamente à magnitude dos coeficientes, ou seja, quanto mais escuro, piores são os valores. Os coeficientes nos mapas foram divididos em quintis.

A análise de dependência espacial foi executada utilizando-se o índice de Moran Global que estima a autocorrelação espacial, podendo variar entre -1 e +1 , além de fornecer a significância estatística do mesmo (valor de p). Após a análise geral foi avaliada a presença de clusters, por meio do Moran local (Local Indicators of Spatial Association - LISA). Para tanto, foram construídos os BoxMap e MoranMap relativos às taxas de Mortalidade e Incidência de câncer de boca e orofaríngeo. O BoxMap apresenta os clusters, independente da significância estatística, enquanto o MoranMap apenas aqueles com valor de $\mathrm{p}<0,05$.

Com o software GeoDa 0.9.9.10 (Spatial Analysis Laboratory, University of Illinois, Urbana-Champaign, Estados Unidos) foi realizada a análise bivariada LISA para avaliação da correlação espacial entre as variáveis desfecho (Taxas de Incidência e Mortalidade de Câncer) e as variáveis independentes (Gini e IDH). Assim, pôde-se visualizar a associação linear entre uma variável $\mathrm{x}_{\mathrm{k}}$ no local $\mathrm{i}, \mathrm{x}_{\mathrm{k}} \mathrm{i}$ e a lag espacial correspondente para a outra variável $\mathrm{Wy}_{\mathrm{l}}\left(\mathrm{l}_{\mathrm{kl}}=\mathrm{x}_{\mathrm{k}}{ }^{\prime} \mathrm{W}_{\mathrm{yi}} / \mathrm{n}\right)$. Para tanto, foram construídos os mapas temáticos com cada par de variáveis e verificado a sua significância estatística.

\section{Resultados}

As taxas de incidência de câncer de boca e orofaríngeo distribuídas no mapa do Município de São Paulo demonstram uma maior concentração da doença (Figura 1a) e menores taxas de mortalidade nas áreas mais centrais da cidade (Figura 1b), com Moran Global de 0,226 e 0,337, respectivamente. No mapa da distribuição espacial do IDH (Figura 1c), os índices mais elevados se localizam nos distritos centrais. Da mesma forma, verifica-se uma menor desigualdade de renda, com baixos coeficientes de Gini (Figura 1d). Na avaliação de autocorrelação global foi encontrado um Índice Moran Global de 0,653 para o IDH e 0,479 para o Gini.

$\mathrm{Na}$ análise de clusters das taxas de incidência apresentadas nas Figuras 2a e 2c, verifica-se a presença de um aglomerado baixo-baixo na região sul e alto-alto na central. Nos mapas de mortalidade (Figura 2b e 2d) um aglomerado baixobaixo nos distritos centrais e alto-alto naqueles mais a leste.

No que se refere à analise de correlação espacial, pode-se observar uma fraca correlação negativa entre as taxas de incidência e de mortalidade $(I=-0,1690)$, ou seja, a despeito da alta incidência de câncer de boca e orofaríngeo em alguns distritos, observa-se uma baixa mortalidade (Figura 3). Da mesma maneira, uma baixa correlação entre as taxas de incidência e IDH $(I=0,211)$ foi constatada, com clusters nas regiões periféricas mostrando baixas taxas de incidência e baixos IDH; enquanto nas áreas centrais, altas e baixas incidências com alto IDH (Figura 4a). As taxas de incidência não apresentaram correlação com o Gini ( $I=-0,093$ - Figura 4b). Por outro lado, houve correlação negativa entre as taxas de mortalidade e o IDH ( $I=-0,414-$ Figura 4c), ou seja, quanto menor o IDH maior a mortalidade. As taxas de mortalidade também aumentam ( $I=0,372$ - Figura 4d) quando aumenta a desigualdade de renda (Gini).

\section{Discussão}

A distribuição espacial das taxas de incidência e de mortalidade por câncer de boca e orofaríngeo no Município de São Paulo demonstra uma forte desigualdade no adoecimento e morte por esta patologia, sendo a incidência mais frequente nas áreas centrais da cidade, enquanto a mortalidade nas regiões mais periféricas. 
Figura 1

Distribuição espacial das taxas de incidência (1a) e mortalidade (1b) por câncer de boca e orofaríngeo, Índice de Desenvolvimento Humano - IDH (1c) e índice de Gini (1d), com os respectivos valores de Moran Global e sua significância estatística. Município de São Paulo, Brasil, 1997.

1a) Taxa de incidência

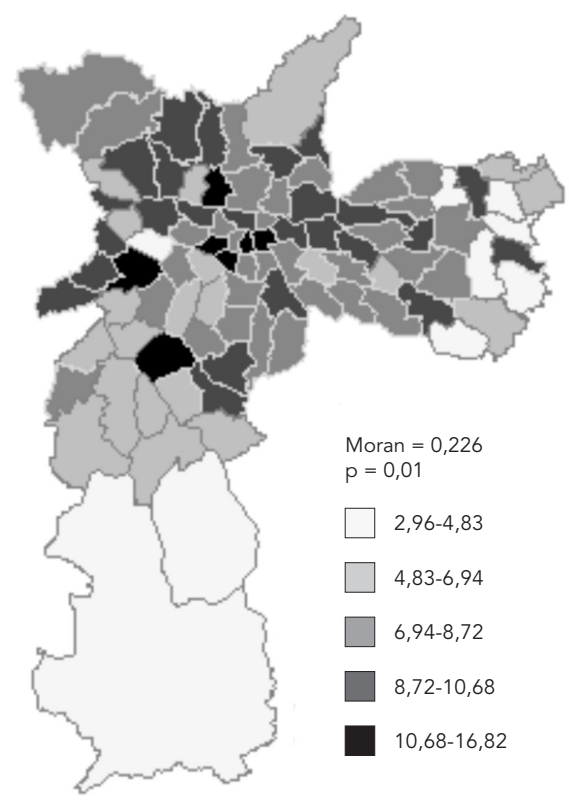

1c) Índice de Desenvolvimento Humano

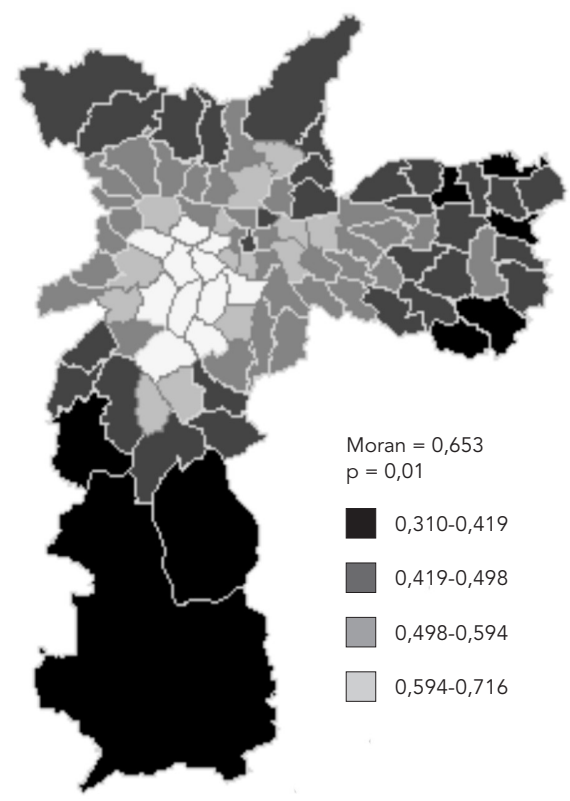

1b) Taxa de mortalidade

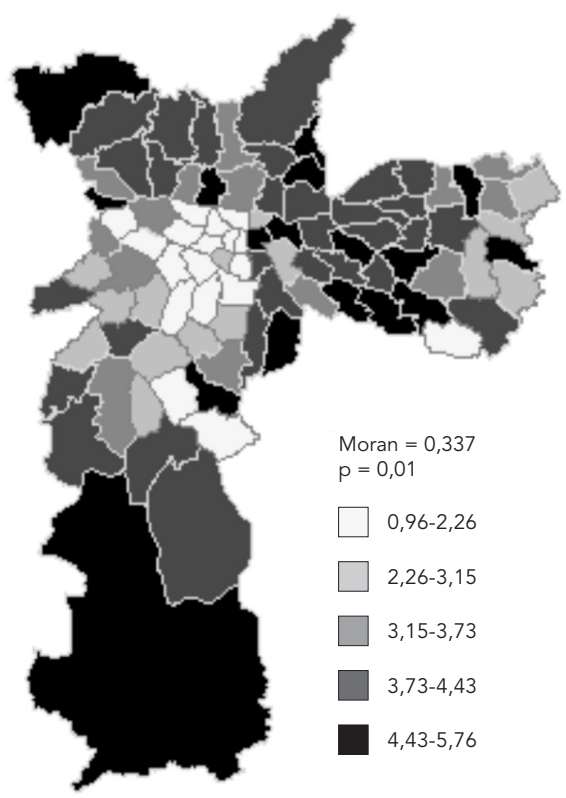

1d) Índice Gini
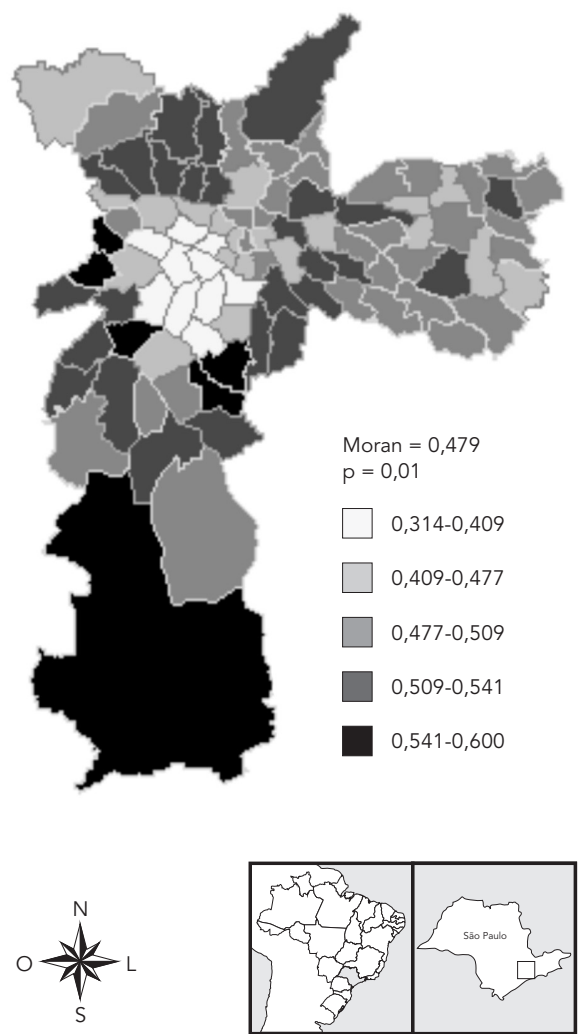


\section{Figura 2}

Distribuição espacial dos clusters das taxas de incidência e mortalidade por câncer de boca e orofaríngeo sem (BoxMap) e com LISA estatisticamente significativo (MoranMap). Município de São Paulo, Brasil, 1997-2008.

2a) Taxa de incidência (BoxMap)

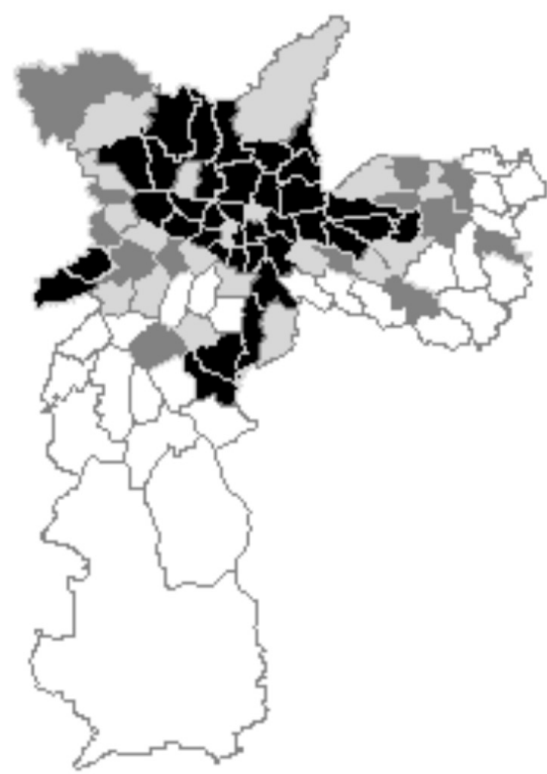

2c) Taxa de incidência (MoranMap)

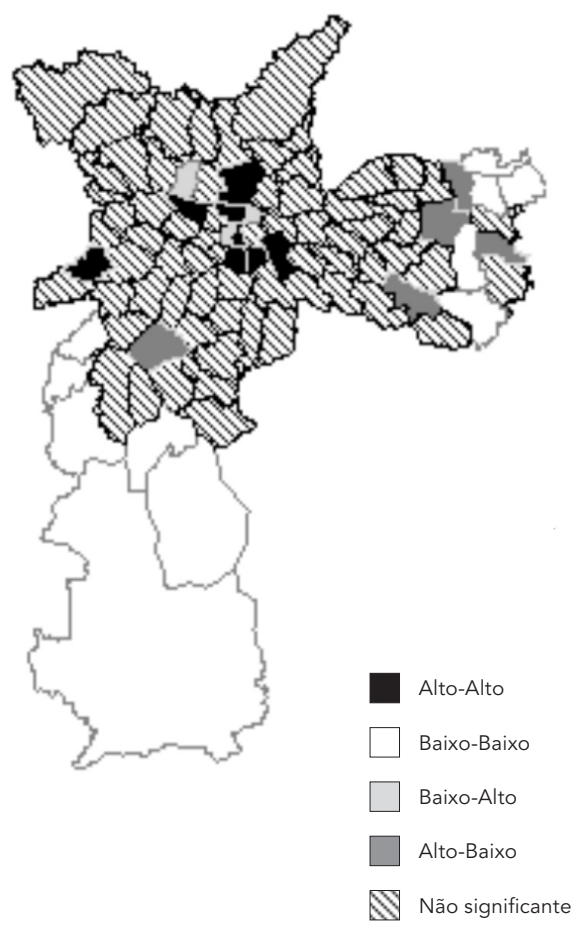

2b) Taxa de mortalidade (BoxMap)

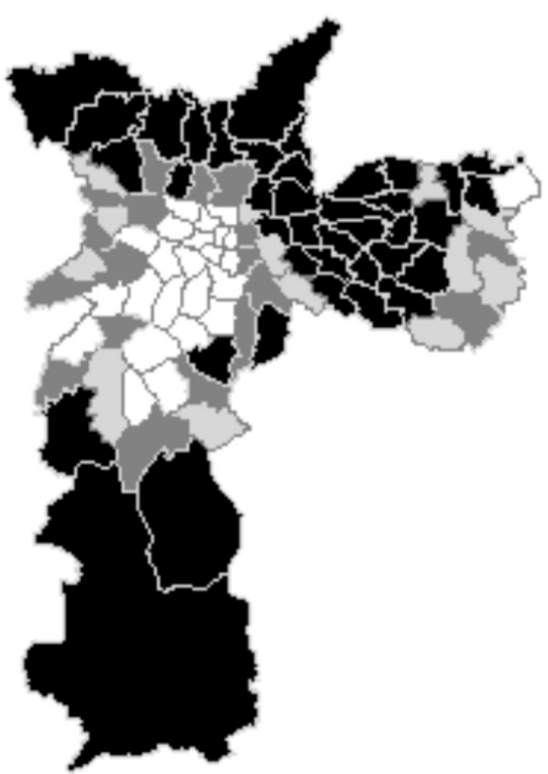

2d) Taxa de mortalidade (MoranMap)

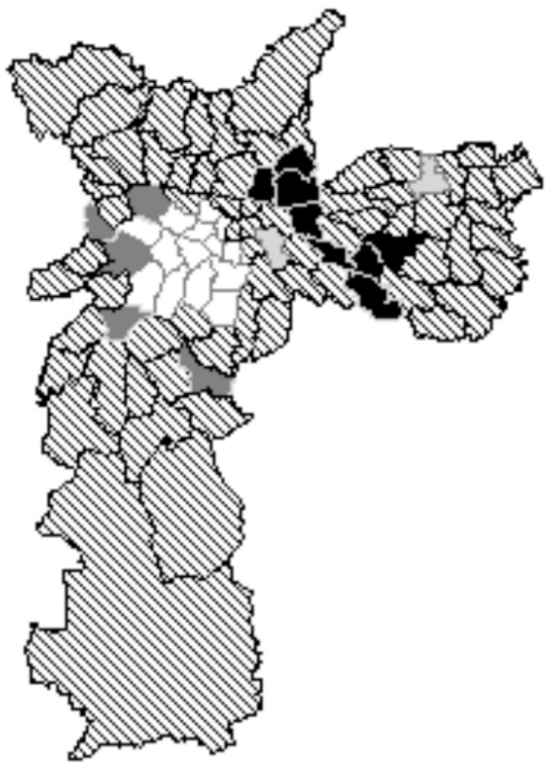

$0 \frac{k_{s}^{N}}{L}$

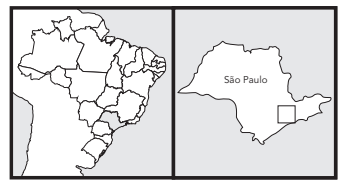


Correlação espacial bivariada LISA entre as taxas de incidência e mortalidade por câncer de boca e orofaríngeo nos distritos do Município de São Paulo, Brasil, 1997-2008.

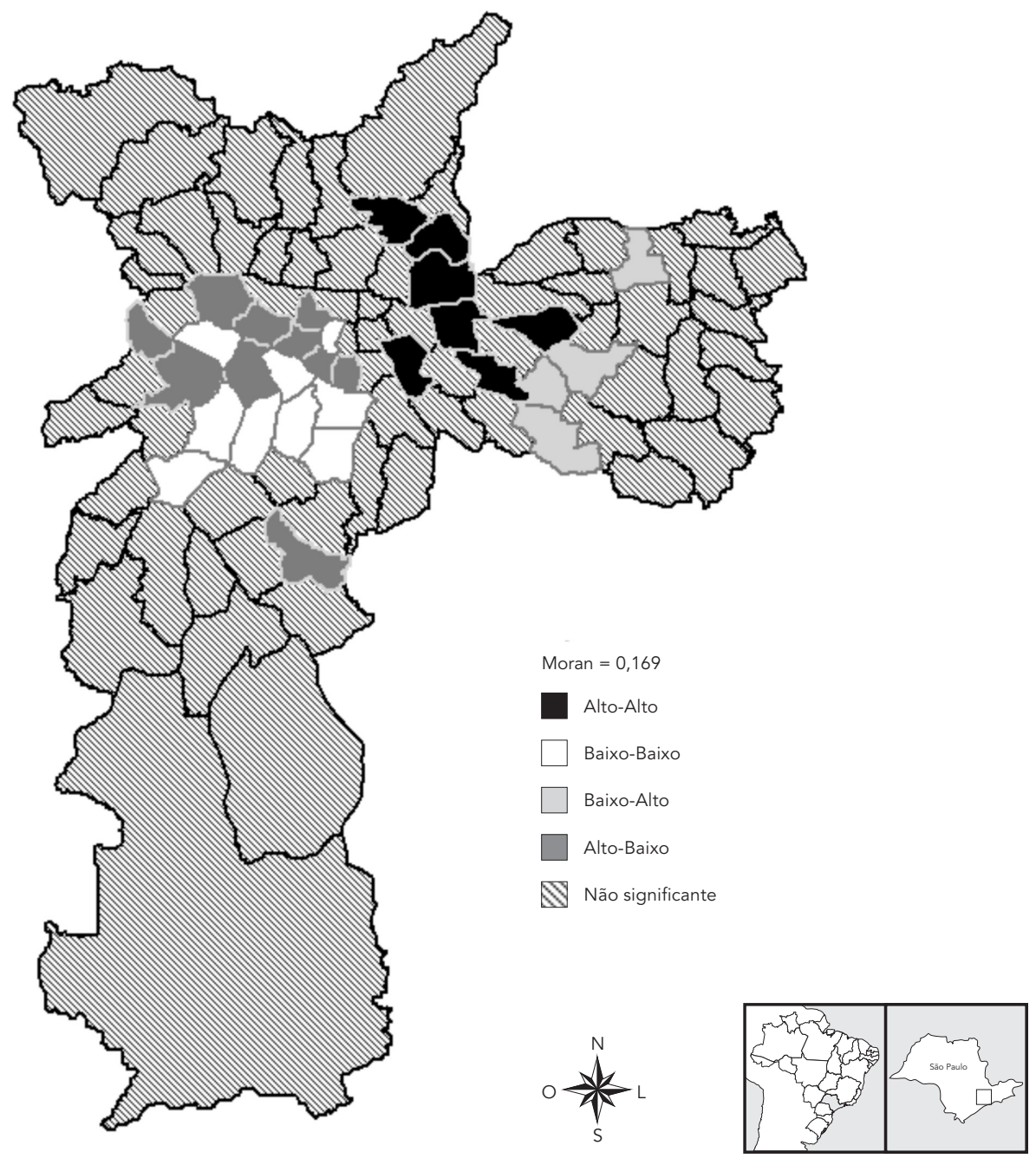

Essa distribuição espacial de mortalidade foi detectada por Antunes et al. 11,12, com taxas de mortalidade obtidas em outros períodos de tempo, ao avaliar a tendência de óbitos por câncer oral na cidade de São Paulo. Ao cruzar os indicadores socioeconômicos de renda, escolaridade, emprego e desigualdade social, os autores constataram uma alta correlação entre os baixos indicadores distritais e aumento na mortalidade, o que coincide com os nossos achados para IDH e Gini.

Contraditoriamente, um estudo realizado por Borges et al. 18 constatou que as capitais bra- sileiras com altas taxas de IDH possuíam maiores taxas de mortalidade. A explicação dos autores foi que a dimensão longevidade do índice teria influenciado esses resultados na medida em que as doenças crônico-degenerativas eram mais comuns em idosos, estrato da população bastante prevalente em metrópoles desenvolvidas. No entanto, o fato das taxas usadas no referido estudo não terem sido padronizadas pela idade parece uma explicação mais plausível para justificar tal distorção.

No que se refere à incidência, apesar da maioria dos casos se distribuir na área mais central, os 
Distribuição dos clusters da correlação espacial bivariada LISA das taxas de incidência e mortalidade por câncer de boca e orofaríngeo com o índice de Desenvolvimento Humano (IDH) e coeficiente de Gini. Município de São Paulo, Brasil, 1997-2008.

4a) Taxa de incidência XIDH

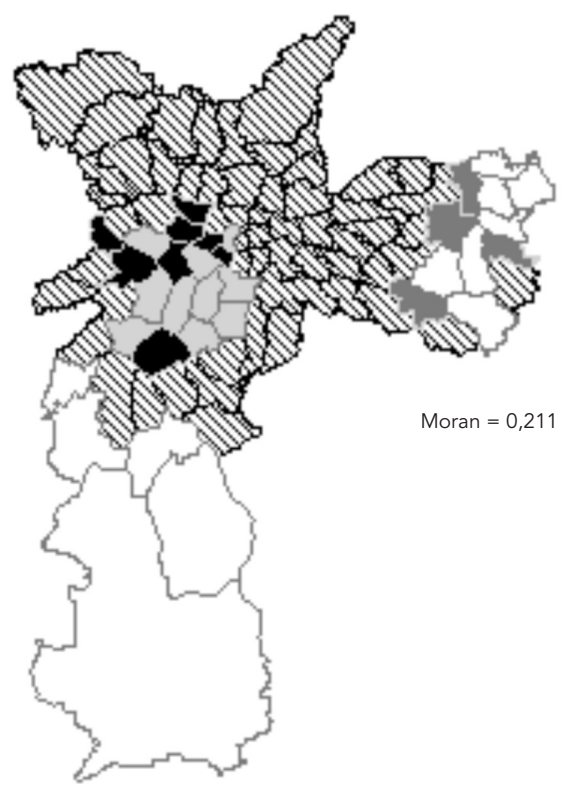

4c) Taxa de incidência $X$ Gini

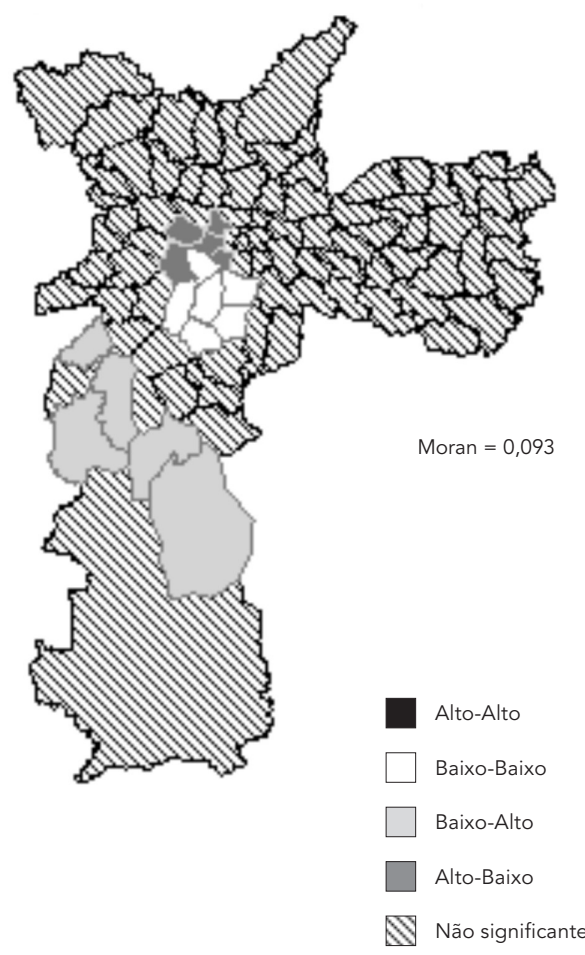

4b) Taxa de mortalidade X IDH

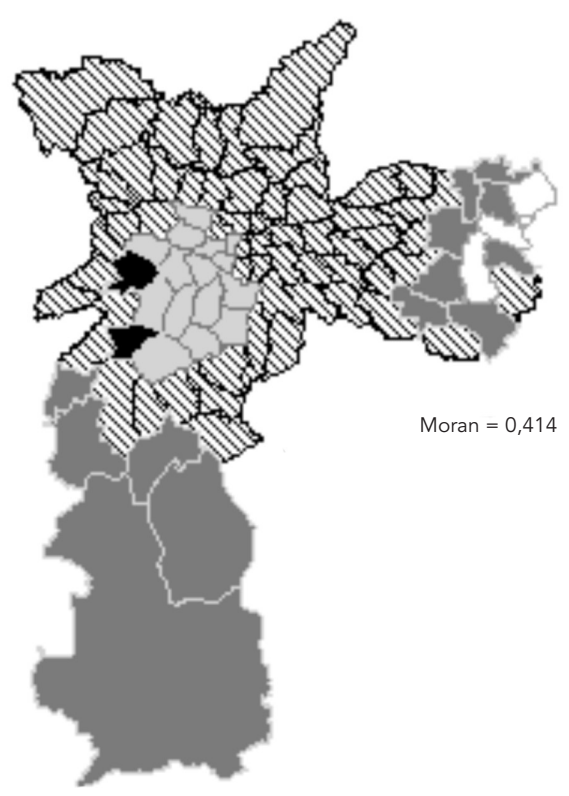

4d) Taxa de mortalidade X Gini
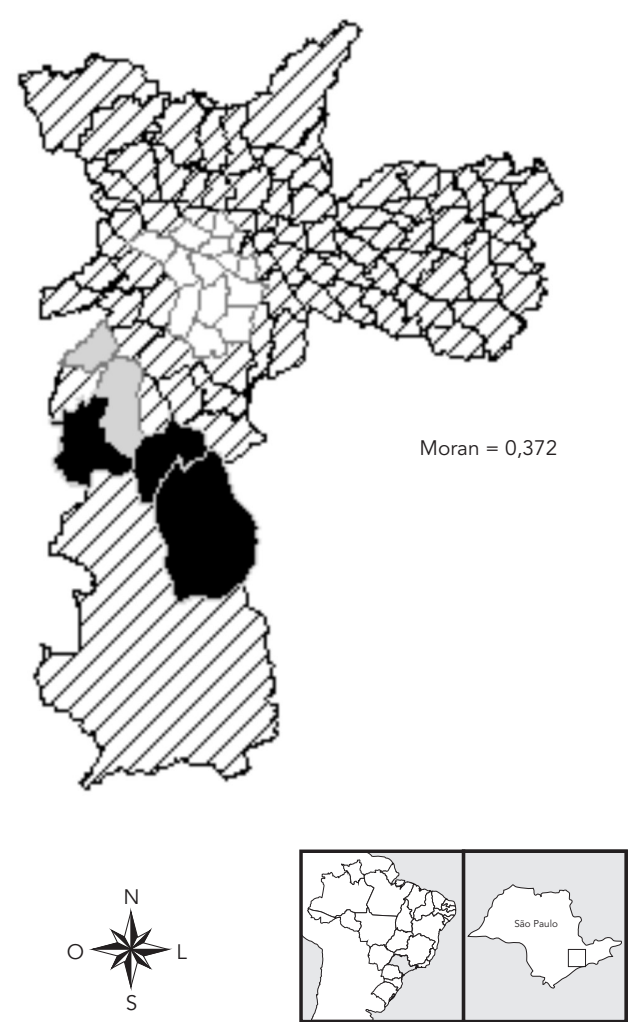
dados não parecem obedecer a nenhum padrão espacial. Isso pode ser confirmado pelos testes de autocorrelação espacial e da correlação bivariada, que apresentaram apenas uma pequena correlação com IDH e nenhuma com a desigualdade de renda nos distritos da cidade. Tais dados contrariam grande parte dos estudos individuados, na medida em que estes demostram haver uma forte associação entre a incidência de câncer de boca e orofaríngeo e as precárias condições socioeconômicas e seus consequentes hábitos deletérios (tabagismo e alcoolismo, principalmente) 10 .

Uma hipótese a ser levantada sobre esse cenário, de maior incidência nas mesmas áreas que apresentam menor mortalidade, seria o acesso que os indivíduos mais abastados teriam aos meios de diagnósticos dos serviços de saúde privados/conveniados. Segundo Pessoto et al. ${ }^{19}$, há uma enorme desigualdade entre possuidores e não possuidores de planos de saúde. De acordo com a Pesquisa de Condições de Vida (PCV) da Fundação Seade 20, 53,5\% dos paulistanos não possuem plano de saúde. Essa desigualdade pode ser explicada pelas diferenças socioeconômicas, seus processos históricos de desenvolvimento e pelas distintas estratégias de expansão do setor suplementar da saúde (convênios e planos). Afinal, a cidade de São Paulo apresenta um dos maiores índices de riqueza e longevidade do Brasil. Ainda, segundo essa pesquisa, os possuidores de planos de saúde demandam, significativamente, mais dos serviços de saúde quando comparados com os não possuidores de planos.

A introdução do espaço como unidade de análise nos estudos epidemiológicos reporta, necessariamente, a uma reflexão acerca da história socioeconômica e política do lugar em estudo. Isso porque o espaço construído e a distribuição da população não têm um papel neutro na vida e na evolução das formações sociais. As diferenças entre os lugares são o resultado do arranjo espacial dos modos de produção particulares, ou seja, as transformações ocorridas no espaço são determinadas pelas necessidades sociais, econômicas e políticas, evoluindo a partir das necessidades do modo de produção e de seus momentos sucessivos, com ampla influência das determinações econômicas internacionais 16 .

São Paulo começa a despontar na história econômica e territorial brasileira no momento em que se instala a era industrial. Essa região já nasce moderna, tanto pelo lado da produção quanto do consumo, como, também, pelo meio ambiente construído, bastante propício às transformações. Esse caráter transformador da cidade para se adequar à modernidade se constitui em uma das explicações para o seu progresso, a despeito do empobrecimento da sua herança cultural do espaço construído. No entanto, essa "modernização incompleta" é bastante seletiva, excluindo grandes parcelas da população dos produtos dessa riqueza 21.

Atualmente, São Paulo é considerada uma "metrópole global”, cujas atividades hegemônicas utilizam a informação como base principal do seu domínio. A acumulação de atividades intelectuais ligadas à nova modernidade assegura a essa cidade a área de numerosas atividades produtivas de ponta. A cidade, considerada um grande centro de decisões econômicas, está presente em todo o território nacional graças a esses nexos geradores de fluxos de informação indispensáveis ao trabalho produtivo. Esse papel se deve às suas atividades quartenárias de criação e controle, pois agora são os fluxos de informação que hierarquizam o sistema urbano 22 .

Essa nova conjuntura econômica da capital paulista tem reflexos significativos na divisão de trabalho, que por sua vez, estabelece uma forte interferência na distribuição das pessoas no espaço. A cada momento da divisão do trabalho, uma nova geografia se estabelece com o objetivo de atender às novas funções, seja construindo novos espaços ou alterando funcionalmente os já existentes. A esse processo pode-se chamar de "geografização da sociedade". Por causa disso se afirma que a pobreza não é apenas criada por causas econômicas, mas, também por causa geográfica. O valor de cada um é ditado pelo lugar onde se encontra 23.

Essa característica de metrópole é peculiar a um país subdesenvolvido industrializado do terceiro mundo, na medida em que a modernidade capitalista é incompleta. Nela convivem a opulência da vida econômica e suas expressões materiais, e os sinais de "desfalecimento", em função do atraso das estruturas sociais e políticas. Daí a designação feita por Santos 22 de "Metrópole, Corporativa e Fragmentada”.

Diante do exposto, pode-se afirmar que São Paulo é a representação máxima do modelo socioeconômico e político implementado no Brasil desde o período colonial português, escravocrata, até o apogeu de seu desenvolvimento industrial, mas sempre se caracterizando como uma sociedade com alto grau de iniquidade social. Um claro exemplo disso é o fato de São Paulo acomodar em seu espaço territorial 1.565 favelas (Portal da Prefeitura Municipal de São Paulo. http://infoci dade.prefeitura.sp.gov.br/htmls/9_distribuicao_ das_favelas_2008_516.html, acessado em 04/ Jul/2011), demonstrando o alto grau de exclusão social dessa importante cidade brasileira.

Nesse sentido, o que mais tem marcado a vida urbana da cidade mais rica do país são os 
contrastes chocantes entre a riqueza de uns poucos e a pobreza de muitos. As disparidades de renda entre as áreas centrais e as periféricas são gritantes. Isso se traduz nos IDH, bem como no índice de Gini. Esse quadro de desigualdade estrutural explica a dificuldade de acesso ao serviço de saúde e o perfil epidemiológico contrastante entre os habitantes da área central da cidade e os da periferia (Fundação Seade. http:/ / wwwseade. gov.br, acessado em 20/Jul/2011).

Os efeitos da desigualdade social sobre os indicadores de saúde têm merecido destaque nas discussões dos estudos da área da saúde. Alguns autores defendem que é a pobreza a maior responsável pelos altos índices de mortalidade e baixa esperança de vida 24 , enquanto outros acreditam que é a desigualdade social per si 25. Isso porque mesmo as sociedades desenvolvidas, com alto PIB (Produto Interno Bruto), não têm melhorado seus indicadores de mortalidade, violência, crime 26 e doenças mentais 27 , o que demonstra os efeitos psicossociais dessa desigualdade influenciando a saúde dos indivíduos.

A despeito dos argumentos de Wilkinson \& Pickett 25 sobre o efeito da desigualdade social e os eventos relativos à saúde, para as doenças crônicas degenerativas como o câncer essa influência ainda não está suficientemente esclarecida. Além disso, há uma grande dificuldade em se encontrar indicadores que contemplem o conceito de classe social e sua real influência sobre a saúde, visto que a maioria deles é baseada na renda e capacidade de consumo dos indivíduos, não sendo o mais adequado para medir as condições de saúde 28 . Nesse sentido, estudos que contemplem essa perspectiva psicossocial das doenças devem ser estimulados para se esclarecer adequadamente os determinantes contextuais do câncer de boca e orofaríngeo.

Retornando à análise sobre o modelo de desenvolvimento implantado no Município de São Paulo, deve-se considerar aqui o papel do Estado. O Estado assume um papel fundamental como indutor desse desenvolvimento, direcionando as suas ações para que os "modernizadores" possam se instalar e operar. Toda a estrutura dos gastos públicos é orientada para assegurar a infraestrutura, transportes ou bens públicos, os quais servem mais, melhor e mais barato às empresas que à população. Isso sem falar nos subsídios diretos, como isenção de impostos, baixas taxas de juros, exportação protegida e financiada, investimento em infraestrutura, pesquisa etc.

Em função disso, as políticas públicas no campo da saúde são direcionadas para atender às camadas sociais hegemônicas em detrimento das mais pobres (hegemonizados), mesmo quando se trata de serviços essenciais. Em São Paulo, a distribuição dos serviços de saúde, particularmente os oncológicos, desfavorece enormemente os habitantes dos distritos periféricos, visto que a grande maioria dos hospitais especializados se concentra nas áreas centrais da cidade (Ministério da Saúde. Cadastro nacional de estabelecimentos de saúde. http://www2.datasus.gov.br/ DATASUS/index, acessado em 25/Jul/2011).

É importante saber onde estão localizados os equipamentos e onde se encontra a população que deles precisam. É preciso cuidar do lugar onde a população vive e lhe dar condição de mobilidade. A imobilidade, que afeta boa parte de nossa população, é sem dúvida uma das principais causas da acessibilidade. O lugar e a mobilidade são elementos constitutivos da cidadania. Como compreender, senão considerando o lugar, o fato de que onde há maior taxa de incidência do câncer não é onde há maior taxa de mortalidade? Entender a saúde em sua complexidade demanda a análise do território. O problema da saúde não se resolve apenas com Unidades de Pronto Atendimento (UPA), Estratégia Saúde da Família (ESF), médicos, farmácias populares.

Em um país como o Brasil, onde os indivíduos de baixa renda apresentam uma maior necessidade de consumo de cuidados médicos e são excluídos dos benefícios dos seguros privados de saúde 19, a dificuldade de acesso aos serviços públicos implica os diagnósticos tardios da doença e precariedade no tratamento. A consequência disso é a morte precoce e evitável das pessoas mais carentes da população. Essa dificuldade de acesso foi verificada por Fonseca et al. 29 em um hospital da capital paulista, ao constatar que a maioria dos pacientes atendidos pelo SUS levava bastante tempo para conseguir atendimento de quimioterapia, além de haver várias interrupções por falta dos quimioterápicos.

Até mesmo no atendimento ambulatorial, em que o nível de complexidade do atendimento é menor, verifica-se iniquidade no acesso aos exames utilizados para prevenção do câncer. Um exemplo disso é a prevenção de câncer de mama. Amorim et al. 31 , ao estudarem os fatores associados a não realização da mamografia e do exame clínico das mamas, constataram que apenas um pequeno número de mamografias e exames clínicos anuais era oferecido pelo SUS. Outros estudos com esse mesmo objetivo foram desenvolvidos em várias partes do país, apresentando resultados bastante semelhantes 31,32 .

Finalmente, com base nos dados encontrados e a despeito das limitações deste estudo, pode-se concluir que existem diferenças espaciais e socioeconômicas no perfil epidemiológico de incidência e, principalmente, no de mortalidade por câncer de boca e orofaríngeo no Município 
de São Paulo. Nesse sentido, parece lícito afirmar que apenas com a implantação de políticas públicas direcionadas à diminuição das iniquidades sociais e de acesso à prevenção primária, ao diagnóstico precoce e a tratamentos de reabilitação será possível reduzir as taxas dessa doença. E como já afirmava Santos 23, tais ações

\section{Resumo}

A ocupação do espaço geográfico é determinada historicamente pelo modelo socioeconômico e pelo dinamismo de suas relações sociais, políticas e ideológicas. O objetivo deste trabalho é avaliar a distribuição espacial e o efeito de indicadores socioeconômicos no adoecimento e morte por câncer de boca e orofaríngeo no Município de São Paulo, Brasil, no período de 1997 a 2008. Os dados foram coletados no Registro de Câncer de Base Populacional e no Programa de Aprimoramento das Informações de Mortalidade - PRO-AIM e georreferenciados pelos softwares Terraview e GeoDa. $O$ referencial teórico para avaliação dos resultados foi baseado na teoria de Milton Santos. As taxas de incidência apresentaram um índice de autocorrelação Global de Moran de 0,226 e as taxas de mortalidade de 0,337. A Incidência de câncer de boca e orofaríngeo não apresenta um padrão espacial bem definido no Município de São Paulo, mas é bastante desigual no que se refere à Mortalidade, concentrando as suas menores taxas na área central, mais rica e economicamente menos desigual.

Neoplasias Orofaríngeas; Iniquidade Social; Geografia

\section{Colaboradores}

M. A. F. Ferreira contribuiu na concepção, análise e interpretação dos resultados e redação do artigo. M. N. Gomes, F. A. S. Michels e A. A. Dantas colaboraram na análise e interpretação dos resultados. M. R. D. O. Latorre participou da revisão crítica do artigo e aprovação final da versão a ser publicada.

\section{Agradecimentos}

À CAPES, por meio do PROCAD, que possibilitou o estágio pós-doutoral do autor. só serão efetivadas quando a cidade, a partir do "meio ambiente construído" por essa diversidade de classes sociais, das diferenças de renda e dos modelos culturais, resistir à difusão dessa racionalidade triunfante do modelo econômico vigente.

\section{Referências}

1. World Health Organization. European health for all database. Copenhagen: World Health Organization Regional Office for Europe; 2005.

2. Wünsch Filho V. The epidemiology of oral and pharynx cancer in Brazil. Oral Oncol 2002; 38: 737-46.

3. Franceschi S, Bidoli S, Herrero R, Muñoz N. Comparison of cancers of the oral cavity and pharynx worldwide: etiological clues. Oral Oncol 2000; 36:106-15.

4. Curado MP, Edwards B, Shin HR, Storm H, Ferlay $\mathrm{J}$, Heanue M, et al. Cancer incidence in five continents. v. IX. Lyon: IARC Scientific Publications; 2007.

5. Shiu MN, Chen THI. Impact of betel quid, tobacco and alcohol on three-stage disease natural history of oral leukoplakia and cancer: implication for prevention of oral cancer. Eur J Cancer Prev 2004; 13:39-45.

6. Li Y, Yang H, Cao J. Association between alcohol consumption and cancers in the Chinese population-a systematic review and meta-analysis. PLoS One 2011; 6:e18776.

7. Lucenteforte E, Garavello W, Bosetti C, La Vecchia C. Dietary factors and oral and pharyngeal cancer risk. Oral Oncol 2009; 45:461-7.

8. Syrjänen S, Lodi G, von Bültzingslöwen I, Aliko A, Arduino P, Campisi G, et al. Human papillomaviruses in oral carcinoma and oral potentially malignant disorders: a systematic review. Oral Dis 2011; 17 Suppl 1:58-72.

9. Rethman MP, Carpenter W, Cohen EE, Epstein J, Evans CA, Flaitz CM, et al. Evidence-based clinical recommendations regarding screening for oral squamous cell carcinomas. J Am Dent Assoc 2010; 141:509-20. 
10. Conway DI, Petticrew M, Marlborough H, Berthiller J, Hashibe M, Macpherson LM. Socioeconomic inequalities and oral cancer risk: a systematic review and meta-analysis of case-control studies. Int J Cancer 2008; 122:2811-9.

11. Antunes JLF, Biazevic MGH, Araújo ME, Tomita NE, Chinellato LEM, Narvai PC. Trends and spatial distribution of oral câncer mortality in São Paulo, Brazil, 1980-1998. Oral Oncol 2001; 37:345-50.

12. Antunes JLF, Borrell C, Pérez G, Boing AF, WünschFilho V. Inequalities in mortality of men by oral and pharyngeal cancer in Barcelona, Spain and São Paulo, Brazil, 1995-2003. Int J Equity Health 2008; 7:14.

13. de Camargo Cancela M, Voti L, Guerra-Yi M, Chapuis F, Mazuir M, Curado MP. Oral cavity cancer in developed and in developing countries: population-based incidence. Head Neck 2010; 32:357-67.

14. Monken M, Barcellos C. Vigilância em saúde e território utilizado: possibilidades teóricas e metodológicas. Cad Saúde Pública 2005; 21:898-906.

15. Durkheim E. As regras do método sociológico. São Paulo: Editora Martin Claret; 2002.

16. Santos M. Da totalidade ao lugar. São Paulo: Edusp; 2008.

17. Ahmad OB, Boschi-Pinto C, Lopez AD, Murray CJL, Lozano R, Inoue M. Age standardization of rates: a new who standard. http://www.who.int/healthinfo/paper31.pdf (acessado em 20/Jul/2011).

18. Borges DM, Fernandes MS, Ferreira MAF, Roncalli AG. Mortalidade por câncer de boca e condição socio-econômica no Brasil. Cad Saúde Pública 2009; 25:321-7.

19. Pessoto UC, Heimann LS, Boaretto RC, Castro IEN, Kayano J, Ibanhes LC, et al. Desigualdades no acesso e utilização dos serviços de saúde na Região Metropolitana de São Paulo. Ciênc Saúde Coletiva 2007; 12:351-62.

20. Costa OV. Pesquisa de condições de vida. São Paulo Perspect 2003; 17:142-50.

21. Santos M. Metrópole corporativa fragmentada: o caso de São Paulo. 2a Ed. São Paulo: Edusp; 2009.

22. Santos M. Por uma economia política da cidade: o caso de São Paulo. 2a Ed. São Paulo: Edusp; 2009.
23. Santos M. Técnica, espaço, tempo: globalização e meio técnico-científico-informacional. 5a Ed. São Paulo: Edusp; 2008.

24. Lynch J, Smith GD, Harper S, Hillemeier M, Ross N, Kaplan GA, et al. Is income inequality a determinant of population health? Part 1. A systematic review. Milbank Q 2004; 82:5-99.

25. Wilkinson RG, Pickett KE. Income inequality and population health: a review and explanation of the evidence. Soc Sci Med 2006; 62:1768-84.

26. Wilkinson RG, Kawachi I, Kennedy BP. Mortality, the social environment, crime and violence. Sociol Health lln 1998; 20:578-97.

27. Pickett KE, James OW, Wilkinson RG. Income inequality and the prevalence of mental illness: a preliminary international analysis. J Epidemiol Community Health 2006; 60:646-7.

28. Ferreira MAF, Latorre MRDO. Desigualdade social e os estudos epidemiológicos: uma reflexão. Ciênc Saúde Coletiva; http://www.cienciaesaudecoleti va.com.br/artigos/artigo_int.php?id_artigo=7964 (acessado em 25/Jul/2011).

29. Fonseca SM, Gutiérrez MGR, Adami NP. Avaliação da satisfação de pacientes oncológicos com atendimento recebido durante o tratamento antineoplásico ambulatorial. Rev Bras Enferm 2006; 59:656-60.

30. Amorim VMSL, Barros MBA, César MBA, Carandina L, Goldbaum M. Fatores associados a não realização da mamografia e do exame clínico das mamas: um estudo de base populacional em Campinas, São Paulo, Brasil. Cad Saúde Pública 2008; 24:2623-32.

31. Dias-da-Costa JS, Olinto MTA, Bassani D, Marchionatti CRE, Bairros FS, Oliveira MLP, et al. Desigualdades na realização do exame clínico de mama em São Leopoldo, Rio Grande do Sul, Brasil. Cad Saúde Pública 2007; 23:1603-12.

32. Lima-Costa MF, Matos DL. Prevalência e fatores associados à realização da mamografia na faixa etária de 50-69 anos: um estudo baseado na Pesquisa Nacional por Amostra de Domicílios (2003). Cad Saúde Pública 2007; 23:1665-73.

Submetido em 23/Set/2011

Versão final reapresentada em 09/Abr/2012

Aprovado em 18/Jun/2012 